\title{
DYNAMIC OPTIMISATION OF ALTERNATING ACTIVATED SLUDGE PROCESSES
}

\author{
M. Fikar*†, B. Chachuat*, M. A. Latif* \\ * Laboratoire des Sciences du Génie Chimique, CNRS-ENSIC, B.P. 451, 1 rue Grandville, 54001 Nancy Cedex, France, Fax. \\ +33 (0)3 831753 26, e-mail: \{latifi,chachuat\}@ensic.u-nancy.fr \\ $\dagger$ Department of Process Control and Information Technology, FCHFT STU, Radlinského 9, 81237 Bratislava, Slovakia, \\ e-mail: fikar@cvt.stuba.sk, Fax: +421 (0)2 396469
}

Keywords: wastewater treatment, activated sludge process, dynamic optimisation.

\begin{abstract}
The study presents dynamic optimisation of a small size single basin wastewater treatment plant. The objectives are to determine an optimal sequence of aeration/non-aeration times and the corresponding periodical steady state subject to minimisation of energy consumption.

Based on the optimal trajectories, two simple feedback rules are proposed that follow from properties of the optimal solution. Simulation results with these simple rules show very satisfactory control performance.
\end{abstract}

\section{Introduction}

A widely used system for biological wastewater treatment is the activated sludge process (ASP). The removal of nitrogen (N) requires two biological processes : nitrification and denitrification. The former takes place under aerobic conditions, whereas the latter requires anoxic environment. For small size plants, i.e. less than 20,000 p.e. (population equivalent), the two processes are very often carried out in a single basin using surface turbines. The nitrification process (respectively denitrification process) is realised by simply switching the turbines on (respectively off).

Control of the ASP has been the subject of a large number of research studies. More control oriented are for example the works of [9] that use simplified linearised model with aeration time as the manipulated variable, [12] have employed interpolating Model Predictive Control, or [10] has used linear multivariable LQ control.

The studies that investigate the problem from the process point of view include for example the works of Isaacs $[8,16]$. A comparison of several control strategies has been investigated in [11].

The aim of this work is to determine an optimal duration of the aeration and non-aeration sequences which will minimise the operation costs as well as satisfy the constraints specified by the EU directives. Based on the analysis of the optimal operational policy, simple feedback rules will be formulated [5].

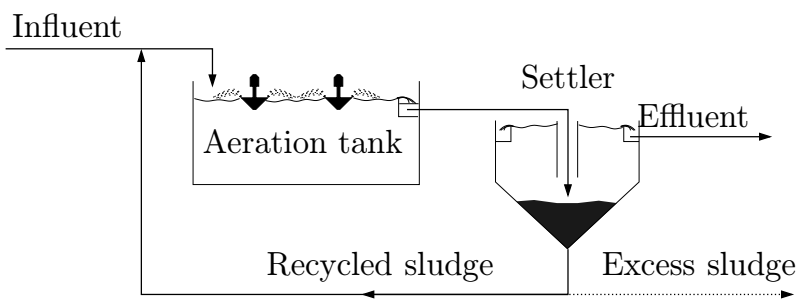

Figure 1: Typical small-size activated sludge treatment plant.

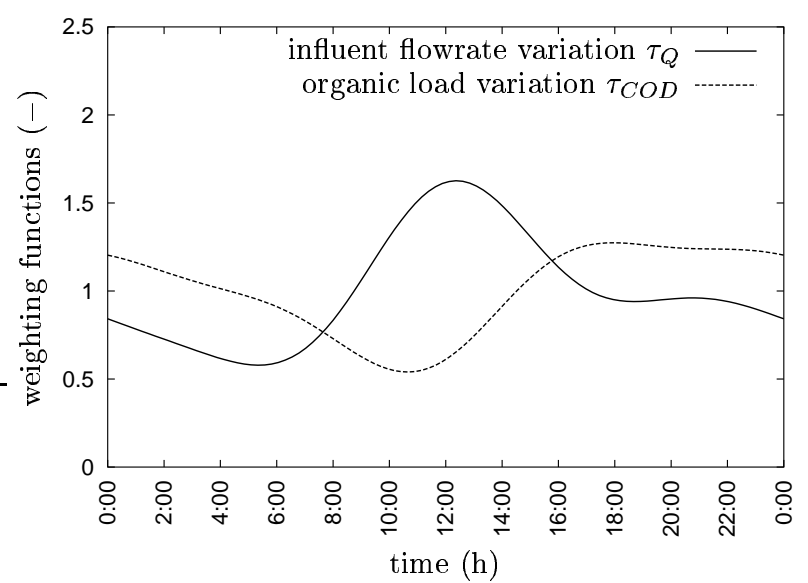

Figure 2: Influent flowrate and organic load variations.

\section{Wastewater Treatment Plant Model}

\subsection{Process}

The process considered is a real small-size treatment plant which is designed for 15,000 p.e. It consists of a unique aeration tank $\left(V^{\mathrm{br}}=2,050 \mathrm{~m}^{3}\right)$ equipped with mechanical surface aerators (turbines) which provide oxygen $(\mathcal{P}=$ $30 \mathrm{~kW}, k_{L} a=4.5 \mathrm{~h}^{-1}$ ) and mix the incoming wastewater with biomass (Fig. 1). The settler is a cylindrical tank where the solids are either recirculated to the aeration tank $\left(Q^{\mathrm{rs}}=7,600 \mathrm{~m}^{3} /\right.$ day $)$ or extracted from the system $\left(Q^{\mathrm{w}}=\right.$ $75 \mathrm{~m}^{3} /$ day).

The influent average flow $Q^{\text {in }}$ is about $3,050 \mathrm{~m}^{3} /$ day and average organic $\mathrm{COD}^{\text {in }}$ and total nitrogen $\mathrm{TN}^{\text {in }}$ loads are $343 \mathrm{mg} / \mathrm{L}$ and $33 \mathrm{mg} / \mathrm{L}$, respectively (after primary treatment). The daily variations of dry weather conditions are based on measured 
data from the plant. It is accounted for by defining weighting functions for both influent flowrate and organic load variations, $\tau_{Q}(t)$ and $\tau_{\mathrm{COD}}(t)$ (Fig. 2).

The average wastewater composition is shown in Table 1. The fractions $f$ are related to the state variables specified below and defined as the ratio between the corresponding concentration and $\mathrm{COD}^{\text {in }}$ or $\mathrm{TN}^{\text {in }}$.

Table 1: Average inlet composition

\begin{tabular}{|c|c|c|c|}
\hline \multicolumn{2}{|c|}{$\mathrm{COD}^{\text {in }}$ fractions } & \multicolumn{2}{|c|}{$\mathrm{TN}^{\text {in }}$ fractions } \\
\hline$f_{\mathrm{SI}}$ & $5 \%$ & $f_{\mathrm{SNH}}$ & $66 \%$ \\
\hline$f_{\mathrm{SS}}$ & $35 \%$ & $f_{\mathrm{SNO}}$ & $0 \%$ \\
\hline$f_{\mathrm{XI}}$ & $10 \%$ & $f_{\mathrm{SND}}$ & $2 \%$ \\
\hline$f_{\mathrm{XS}}$ & $35 \%$ & $f_{\mathrm{XND}}$ & $32 \%$ \\
\hline$f_{\mathrm{XBH}}$ & $15 \%$ & & \\
\hline$f_{\mathrm{XBA}}$ & $0 \%$ & & \\
\hline
\end{tabular}

\subsection{Model}

The model used in this work is based on the Activated Sludge Model No.1 (ASM 1) by [7]. This is the most popular mathematical description of the biochemical processes in the reactors for nitrogen and chemical oxygen demand (COD) removal. It was adopted with two modifications: (i) the state variable describing the total alkalinity is not included, and (ii) inert particulate material from influent and from biomass decay are combined into a single variable $\left(X_{\mathrm{I}}\right)$ since they are of minor interest. The resulting biodegradation model consists of 11 state variables $\left(S_{\mathrm{I}} S_{\mathrm{S}} X_{\mathrm{I}} X_{\mathrm{S}} X_{\mathrm{B}, \mathrm{H}}\right.$

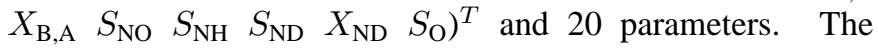
kinetic and stoichiometric parameter values considered are those defined for the simulation benchmark [1]. The complete set of equations, parameters values, and influent conditions can be found on the European COST action 624 website (http: / / www.ensic.u-nancy. fr/COSTWWTP) as well as from the authors.

The model assumptions are (i) perfect mixing in the reactor, (ii) perfect separation of liquid and solid phases in the settler, (iii) the sum of all settler flowrates equals the settler influent flowrate.

The model differential equations can be stated as

$$
\dot{\boldsymbol{x}}=\boldsymbol{f}(\boldsymbol{x})
$$

where $f$ are right hand sides of the differential equations given by:

- For soluble components $(i=1,2,7,8,9)$ :

$$
f_{i}(\boldsymbol{x})=\frac{Q^{\mathrm{in}}\left(x_{i}^{\mathrm{in}}-x_{i}\right)}{V^{\mathrm{br}}}+r_{i}(\boldsymbol{x})
$$

- For particulate components $(i=3,4,5,6,10)$ :

$$
f_{i}(\boldsymbol{x})=\frac{\left[Q^{\mathrm{in}}\left(x_{i}^{\mathrm{in}}-x_{i}\right)+Q^{\mathrm{rs}} \frac{Q^{\mathrm{in}}-Q^{\mathrm{w}}}{Q^{\mathrm{rs}}+Q^{\mathrm{w}}} x_{i}\right]}{V^{\mathrm{br}}}+r_{i}(\boldsymbol{x})
$$

- For dissolved oxygen concentration $(i=11)$ :

$$
\begin{aligned}
f_{11}^{(1)}(\boldsymbol{x}) & =f_{11}^{(2)}(\boldsymbol{x})+u_{b} \mathcal{A}_{\mathrm{O}} \\
f_{11}^{(2)}(\boldsymbol{x}) & =\frac{Q^{\text {in }}\left(x_{11}^{\mathrm{in}}-x_{11}\right)}{V^{\mathrm{br}}}+r_{11}
\end{aligned}
$$

where $r_{i}(\boldsymbol{x}), i=1, \ldots, 11$ represents the apparent reaction rate depending on the kinetic rates of degradation of the components.

As we can see, the mass balance equation related to the concentration of dissolved oxygen may contain an additional term $\mathcal{A}_{\mathrm{O}}$ which describes the oxygen transfer from the turbines:

$$
\mathcal{A}_{\mathrm{O}}=k_{L} a\left(S_{\mathrm{O}}^{\max }-S_{\mathrm{O}}\right)
$$

where $k_{L} a$ is the oxygen transfer coefficient and $S_{\mathrm{O}}^{\max }$ is the dissolved oxygen saturation concentration $\left(S_{\mathrm{O}}^{\max }=10 \mathrm{mg} / \mathrm{L}\right)$.

The input $u_{b}$ is a binary sequence switching between 1 and 0 and represents the state of turbines (on/off) that aerate the plant. Without loss of generality, it is assumed that at time $t=0$ the turbines are on.

\subsection{Control Variables}

Although there are more choices for manipulated variables, practically only the sequence of switching times (when the turbines switch on/off) influences the operation of the wastewater treatment plant (WWTP).

Unfortunately, this control variable does not occur explicitly in the model equations. However, it is possible to normalise the model with respect to time in order to obtain an alternative description where the true manipulated variables occur in the system equations. Let us assume that there are $N_{c}$ cycles within one day consisting of a period of aeration followed by a period of non-aeration and let us denote the lengths of the finite time elements by $\Delta t_{1}, \ldots, \Delta t_{2 N_{c}}$. The aim of the normalisation is to change the time intervals $\Delta t_{1}, \Delta t_{1}+\Delta t_{2}, \ldots, T$ into evenly spaced fixed time intervals $1 / 2 N_{c}, 2 / 2 N_{c}, \ldots, 1$. This results in the modified system equations

$$
\frac{\mathrm{d} \dot{\boldsymbol{x}}}{\mathrm{d} \tau}=u(\tau) \boldsymbol{f}\left(\boldsymbol{x}, u_{b}\right), \quad \tau \in[0,1]
$$

where $u(\tau)$ is a piece-wise constant sequence of the length $2 N_{c}$ containing the switching times $\Delta t_{1}, \ldots, \Delta t_{2 N_{c}}$.

\section{Optimisation Problem}

The optimisation problem can be stated as follows:

$$
\min _{\boldsymbol{u}, \boldsymbol{p}} J=\frac{\sum_{j=1}^{N_{c}} u\left(\tau=\frac{2 j-1}{2 N_{c}}\right)}{T}
$$


subject to

$$
\begin{aligned}
\frac{\mathrm{d} \dot{\boldsymbol{x}}}{\mathrm{d} \tau} & =u \boldsymbol{f}\left(\boldsymbol{x}, u_{b}\right), \quad \tau \in[0,1] \\
\boldsymbol{x}(0) & =\boldsymbol{p} \\
\varepsilon & >\|\boldsymbol{x}(1)-\boldsymbol{p}\| \\
10 & \geq \operatorname{TN}(\tau) \\
u(j) & \in[15,120] \mathrm{min}, \quad j=1,2 N_{c} \\
T & =\sum_{j=1}^{2 N_{c}} u(j)
\end{aligned}
$$

The explanation is as follows.

In this study, economic cost has been chosen as the most realistic. About $3 / 4$ of the total cost is related to energy consumption of the aeration turbines [14]. As these operate in on/off mode, minimising the time of aeration will decrease the operating costs. Therefore, the dimensionless cost function is defined as (8).

In order to find a stationary regime, initial conditions of the plant are assumed to be unknown and are subject to optimisation. Equation (10) assigns the initial conditions to the optimised vector of parameters $\boldsymbol{p}$ and (11) assures final states are similar as the initial ones ( $\varepsilon$ is chosen as some small positive number) so that the stationary regime is achieved.

Inequality (12) represents constraint on the quality of the effluent water that should not exceed $10 \mathrm{mg} / \mathrm{L}$ of total nitrogen during the whole operation. Inequalities (13) are imposed on the aeration times to ensure the feasibility of the computed aeration profiles and to prevent the turbines from damaging.

The minimum air-on and air-off times are set to 15 minutes to avoid too frequent cycling of the turbines and to ensure that the activated sludge after anoxic periods will be sufficiently aerated and mixed in the aeration tank.

Maximum times of 120 minutes are also considered to prevent floc sedimentation in the aeration tank as well as anaerobic conditions, hence modifying the degradation performances. In addition, the CSTR assumption may be no longer valid for too long non-stirring periods.

Finally, (14) sets the optimisation time equal to one day, because the disturbances are assumed to be periodic within this interval.

There are several possible dynamic optimisation methods that can solve this problem. As the optimised variables are time independent, it is particularly suitable to convert the original dynamic problem into static one. In order to avoid inclusion of integer variables that currently may pose problems and complicate the solution, it is supposed that the number of the cycles $N_{c}$ is known. In this case the problem reduces to nonlinear programming problem (NLP).

In this work, control vector parameterisation approach has been chosen to transform the problem to NLP. As optimisation solver, the general purpose dynamic optimisation package

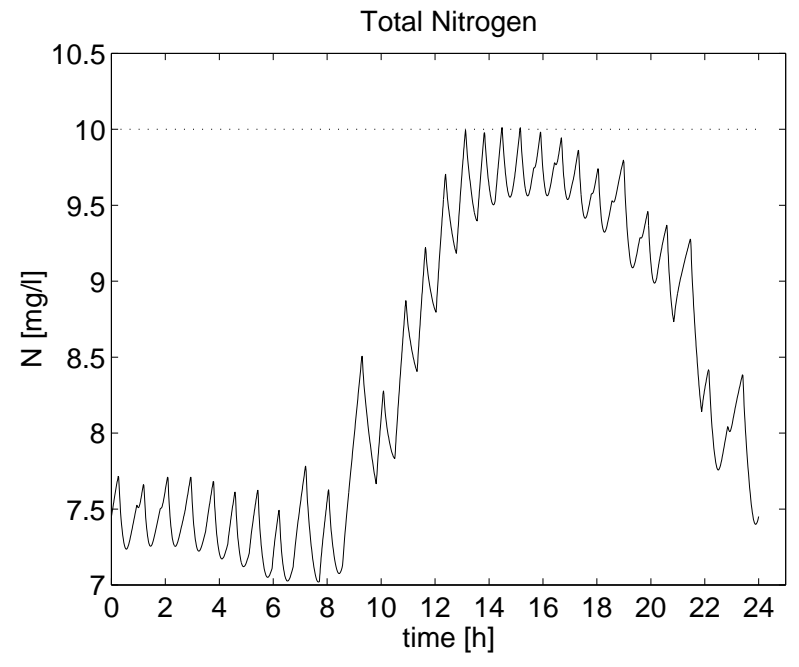

Figure 3: Optimal stationary nitrogen constraint trajectory for $J=39.51 \%$

DYNO [6] has been employed. Its underlying NLP solver is NLPQL [13], IVP solver can be VODE [3] or DASSL [2], and the gradients are calculated via backward integration of the adjoint variables.

\section{Results and Discussion}

Based on some preliminary experiments [4], the number of cycles has been fixed to $N_{c}=29$ and was not a subject of further optimisation as this would lead to mixed integer dynamic optimisation.

The solution of the optimisation problem defined in the preceding section converged to the optimal aeration profile with the average aeration of $39.51 \%$ shown in Fig. 3. It can be noticed that the total nitrogen hits the maximum constraint.

Analysing the optimal state trajectories for all 11 states it has been found that two of them, nitrate and nitrite nitrogen concentration $S_{\mathrm{NO}}$ and dissolved oxygen concentration $S_{\mathrm{O}}$ are are particularly interesting, because on the contrary to other states, only these two show a limited sensitivity towards the disturbations (inlet flowrates and inlet concentrations). More precisely, switching off the turbines in the obtained optimal stationary regime occurs frequently either if the concentration of $S_{\mathrm{NO}}$ falls close to zero or if the concentration of $S_{\mathrm{O}}$ is sufficiently high. Moreover, these two states can be measured. A simple control strategy can then be proposed from their behaviour:

1. Start aeration when $S_{\mathrm{NO}}$ decreases sufficiently close to zero,

2. Stop aeration when $S_{\mathrm{O}}$ reaches a certain value.

We note that the first rule has been proposed for example by [15] for a different alternating WWTP setup (BIODENITRO) and based on an analysis of a simplified ASM1 model. This rule follows from the fact that the denitrification 

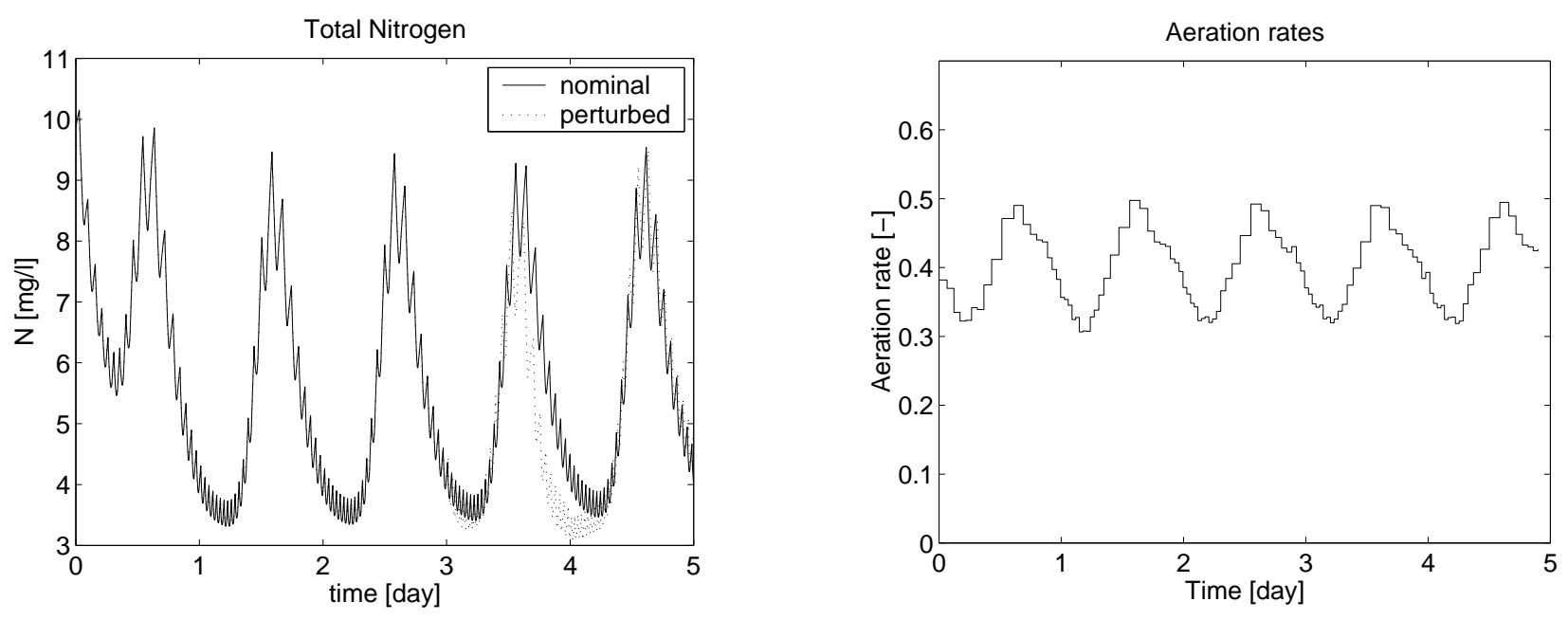

Figure 4: Trajectories for rule based control. Left: Nominal and perturbed nitrogen constraint, right: nominal aeration policy

period should be large enough to consume most of the $S_{\mathrm{NO}}$ in the reactor. On the other hand, it is not necessary to make it longer if the $S_{\mathrm{NO}}$ concentration is already close to zero as no relevant bioprocess takes place in the reactor.

Also the second rule can be found quite often working well in practice. However, it is not quite clear what the $S_{\mathrm{O}}$ setpoint should be.

The application of these simple rules is shown in Fig. 4 where the total nitrogen concentration is shown for two cases. The first one denoted as nominal applies the rules with values of $S_{\mathrm{NO}}(\min )=0.01 \mathrm{mg} / \mathrm{L}$ and $S_{\mathrm{O}}(\max )=0.7 \mathrm{mg} / \mathrm{L}$. In the perturbed case it is supposed that the third day is rainy with $300 \%$ increase of the influent flow and with 50\% decrease of the influent concentrations for the whole day.

It is interesting to note that the simple feedback control policy produces aeration rates that are not constant during the day but act actively against the disturbances. Continuation of the simulation for another 200 days (not shown here) to attain stationary operation gives the average aeration rate approximately the same as that of the optimal control (39.60\%) with the peak concentration of the total nitrogen only slightly higher $(10.74 \mathrm{mg} / \mathrm{L})$.

Also, the disturbance rejection caused by rainy day simulation is satisfactory. However, as rainy conditions have the largest impact on the settler behaviour that has not been modelled here, the more detailed model may behave differently.

\section{Conclusions}

This paper has dealt with the determination of the optimal aeration strategies for small-size activated sludge plants based on dynamic optimisation. The model employed for the optimisation is the standard ASM1 model. From the control point of view, this model represents a system with one manipulated input - sequence of aeration/non-aeration times and with primary output - total nitrogen concentration in the effluent. No setpoint is known for the output, only its upper constraint.
Based on the problem specifications, the optimisation task has been defined and solved using the dynamic optimisation solver DYNO. The time base for the optimisation has been fixed to one day, based on the periodicity of the disturbances - composition and flowrate of the influent. To find an optimal stable operation, the initial states were considered as optimised parameters and a terminal constraint was included that initial and terminal states should be the same.

Based on the optimal stationary state profiles, two simple rules have been defined that relate the start and stop of aeration to nitrate and dissolved oxygen levels, respectively. Application of these simple rules for several days showed only a very small deterioration compared to the dynamic optimisation solution.

The results obtained can serve to several purposes. The dynamic optimisation solution can indicate the relation between the actual and optimum operation and whether there is a room for improvement that will justify additional investments due to necessary sensors needed for state estimation. Next, the stationary profile can be used as a setpoint at the existing plant or the simple rules observed here can be used to enhance the existing operating policies.

\section{Acknowledgments}

This work has been supported by the Ministère de la Recherche et Technologie and Conseil Régional de Lorraine. The first author has also been supported by VEGA MSSR (grants no. $1 / 135 / 03$ and $1 / 8108 / 01$ ). This financial support is very gratefully acknowledged.

\section{References}

[1] J. Alex, J.-F. Beteau, J.B. Copp, C. Hellinga, U. Jeppsson, S. Marsili-Libelli, M.-N. Pons, H. Spanjers, and H. Vanhooren. Benchmark for evaluating control strategies in wastewater treatment plants. In Proc. ECC'99, Karlsruhe, Germany, 1999.

[2] K. E. Brenan, S. E. Campbell, and L. R. Petzold. Nu- 
merical Solution of Initial Value Problems in DifferentialAlgebraic Equations. North-Holland, New York, 1989.

[3] P. N. Brown, G. D. Byrne, and A. C. Hindmarsh. VODE: A variable coefficient ODE solver. SIAM J. Sci. Stat. Comput., 10:1038 - 1051, 1989.

[4] B. Chachuat. Méthodologie d'optimisation dynamique et de commande optimale des petites stations d'épuration à boues activées. $\mathrm{PhD}$ thesis, Institut National Polytechnique de Lorraine, 2001.

[5] D. Chmúrny, R. Prokop, and M. Bakošová. Automatic Control of Technological Processes. Alfa, Bratislava, 1988. (in Slovak).

[6] M. Fikar and M. A. Latifi. User's guide for FORTRAN dynamic optimisation code DYNO. Technical Report mf0201, LSGC CNRS, Nancy, France; STU Bratislava, Slovak Republic, 2001.

[7] M. Henze, C. P. L. Grady, W. Gujer, G. v. R. Marais, and T. Matsuo. Activated Sludge Model No. 1. Technical Report 1, IAWQ, London, 1987.

[8] S. Isaacs. Short horizon control strategies for an alternating activated sludge process. Wat. Sci. Tech., 34(1-2): 203-212, 1996.

[9] Hyunook Kim, T. J. McAvoy, J. S. Anderson, and O. J. Hao. Control of an alternating aerobic-anoxic activated sludge system - Part 2: Optimization using a linearized model. Control Engineering Practice, 8(3):279-289, 2000.

[10] C.-F. Lindberg. Multivariable modeling and control of an activated sludge process. Wat. Sci. Tech., 37(12):149156, 1998.

[11] L. J. S. Lukasse, K. J. Keesman, A. Klapwijk, and G. van Straten. A comparison of NH4/NO3 control strategies for alternating activated sludge processes. Wat. Sci. Tech., 39 (4):93-102, 1999.

[12] S. Joe Qin, V. M. Martínez, and B. A. Foss. An interpolating model predictive control strategy with application to a waste treatment plant. Computers chem. Engng., 21 (S1):S881-S886, 1997.

[13] K. Schittkowski. NLPQL : A FORTRAN subroutine solving constrained nonlinear programming problems. Annals of Operations Research, 5:485-500, 1985.

[14] J.-L. Vasel. Contribution à l'étude des transferts d'oxygène en gestion des eaux. $\mathrm{PhD}$ thesis, Fondation Universitaire Luxemourgeoise, Luxembourg, Arlon, 1988.

[15] H. Zhao, S. H. Isaacs, A. Søeberg, and M. Kümmel. A novel control strategy for improved nitrogen removal in an alternating activated sludge process - Part I. Process analysis. Wat. Res., 28(3):521-534, 1994.
[16] H. Zhao, S. H. Isaacs, H. Søeberg, and M. Kümmel. An analysis of nitrogen removal and control strategies in an alternating activated sludge process. Wat. Res., 29(2): 535-544, 1995. 